\title{
APPENDICITIS IN CHILDHOOD AND SYSTEMIC INFLAMMATORY RESPONSE SYNDROME (SIRS)
}

\author{
Y. Dimcheva, Kr. Kalinova*, D. Dinkov, K. Georgiev, B. Brahomov, E. Kyazimova \\ Department of Pediatric Surgery, Medical Faculty, Trakia University, Stara Zagora, Bulgaria
}

\begin{abstract}
To our knowledge, the prevalence of Systemic Inflammatory Response Syndrome (SIRS) in pediatric patients with appendicitis has not been previously investigated. This study sought to determine the prevalence, test characteristics, and severity of illness of pediatric patient's appendicitis and systemic inflammatory response syndrome (SIRS). Specific aim was to determine the prevalence of SIRS at the time and sings of presentation of pediatric patients with appendicitis. Additionally, we sought to determine if the presence of SIRS had any value in predicting their clinical outcome. The early definition of a systemic inflammatory response syndrome (SIRS) was built upon a foundation of basic clinical and laboratory abnormalities that were readily available in almost all clinical settings.
\end{abstract}

Key words: SIRS, appendicitis,children

The surgical indication for acute appendicitis has been discussed diversely. The tendency to rapid development of the inflammatory process in the child's body and generalization leads to the expression of objectively existing symptom syndrome designated as systemic inflammatory response-SSVO/SIRS/ $(1,2,8)$. This definition was introduced in 1992 by the American College of Chest Physicians (ACCP) and the Society of Critical Care Medicine (SCCM). Causes of the syndrome are nonspecific. It can be due to ischemia, infection, injury, inflammation or a combination of factors.

SIRS, refers to the Systemic Inflammatory Response Syndrome, a stress response defined by abnormalities of temperature, heart rate, respiratory rate and peripheral $\operatorname{WBC}(1,2)$.

Two or more of the following disturbances make the diagnosis of SIRS: Core temperature above 38.5 or below $36 \mathrm{C}$; tachycardia defined as mean heart rate above two standard deviations above normal for age or bradycardia defined as mean heart rate less than the 10th percentile for age; tachypnea defined as mean respiratory rate above two standard deviations for age; leukocyte count elevated or depressed for age or $10 \%$ immature neutrophils. The pediatric group is divided in six clinically and

\footnotetext{
* Correspondence to: Krasimira Kalinova MD, PhD, Stara Zagora 6003 Bulgaria Trakia

University, Medical faculty, Department of

Pediatric surgery, krasimirakalinova@abv.bg
}

physiologically meaningful age groups for agespecific vital sign and laboratory variables to meet SIRS criteria (3-6). There is a mandatory requirement for abnormality of temperature or leukocyte count to be present for a diagnosis of SIRS be considered. Since SIRS plus infection equal sepsis it is crucial to recognize SIRS in its initial phase to start adequate management. Multiple initializing triggers SIRS such as infection, trauma and surgery. Most pediatric cases of SIRS occur in the 2-5 year age group with a median age of 30 months. Severe sepsis refers to acute organ dysfunction or tissue hypoperfusion secondary to infection and septic shock is severe sepsis plus hypotension not reversed with fluid resuscitation. There is a high risk of developing sepsis in children with SIRS. Early SIRS diagnosis eventually leads to early goal-therapy for sepsis.

Pediatric appendicitis that presents initially with SIRS have a higher length of stay and risk incidence of developing intraabdominal abscess. Systemic organ failure and intestinal dysfunction are strong risk factors for postoperative SIRS in children. These preexistingconditions lead to disruption of normal intestinal flora or barrier function predisposingchildren to dramatic SIRS episodes after intestinal surgery.

Pediatric sepsis is generally considered to comprise a spectrum of disorders that result from infection by bacteria or the toxic products of these microorganisms. Early recognition and 
intervention clearly improve outcome for infants and children with conditions that lead to sepsis.

The spectrum of sepsis ranges from microbial invasion of the bloodstream or intoxication with early signs of circulatory compromise/including tachycardia, tachypnea, peripheral vasodilation, and fever (or hypothermia)/to full-blown circulatory collapse with multiple organ dysfunctions syndrome (MODS) and death $(14,15)$.

\section{Signs and symptoms}

Obtain a complete history as part of the evaluation for possible sepsis. Symptoms that may be noted include the following:

- Fever (most common presenting symptom of children with systemic inflammatory response syndrome [SIRS])

- Racing heart

- Rapid or labored breathing

- Cool extremities

- Color changes

- The following should be inquired into:

Exposures to infectious illnesses and other sources of insult

- Activity level

- Mental status (relative to age)

- Urine output

- Immunizations

- Drug allergies

Perform a complete physical examination. Findings reflective of sepsis may include the following:

- Subtle changes in vital signs (often the first indicators of impending SIRS)

- Hypotension, mental status changes, and anuria (late signs)

- Hypothermia (often more ominous than fever)

- Localizing signs of infection

\section{Diagnosis}

The appearance of the syndrome systemic inflammatory response in response to the inflammatory process in the body forms the clinical picture of sepsis.

On Cellular Level tissues are deprived of adequate kravosnablyadavane and suffer oxygen consequently begin to form toxic metabolites and, if not correct the situation leads to severe metabolic dysfunction, which will not allow reconstruction of the tissue is independent of the fact that blood flow in the time to recover.
Cellular dysfunction plays a particularly important role in septic shock, in which the formation of endotoxins triggered inflammatory cascade that results in the formation of cytokines, proinflammatory monokines, prostaglandins, leukotrienes, histamine, proteases and nitrous oxide. These mediators have different regulatory function and influence of different types of receptors. The presence of these mediators resulted in a condition which is known as syndrome of systemic inflammation.

Bacteremia, sepsis and septic shock Infection was defined as "microbial phenomenon with inflammatory response to invasion of microorganisms or normally sterile tissue by these organisms."

Bacteremia is the presence of bacteria in the blood, but this condition does not always lead to sepsis or SIRS. Sepsis is a systemic response to infection, and is defined as the presence of SIRS in addition to the documented or presumed infection. Severe sepsis meets the above criteria and is associated with organ dysfunction, hypoperfusion or hypotension.

Sepsis-induced hypotension, defined as "systolic blood pressure less than $90 \mathrm{~mm} \mathrm{Hg}$ or a reduction of more than $40 \mathrm{~mm} \mathrm{Hg}$ from baseline in the absence of other causes of hypotension." Patients meeting the criteria for septic shock if they have persistent hypotension and perfusion disorders, despite adequate fluid resuscitation, in which the function of the body is unable to maintain homeostasis. Organ dysfunction or refractory hypotension associated with ischemic or inflammatory process, not an infectious etiology. Appendix is a vestigial organ that starts from the rear-medial surface of the coecum and continues medially from it. Rare cases of appendicitis in infants and young children 1 to 3 years, connects with the wider lumen of the appendix and less likely to form etc. "cavite clouse" - participating in the pathogenesis of the disease (8). Inflammation of the appendix / appendicitis / usually takes place in intestinal, lymphogenic, heamatogenous time or in the vicinity and can be associated with various autoimmune local process. Higher incidence of complication with SIRS in childhood is associated with anatomical and physiological characteristics $(7,8)$.

Increased local irritation of the wall of the appendix and lymph leads to blood stasis, followed by edema, favoring the development 
of pathogenic microorganisms, obtained appendicular `s wall and eventually gangrene. The clinical picture of appendicitis begins with the onset of pain in the abdomen, in the beginning which can be epigastral, but after 6 to 12 hours is localized in the ileocecal region. The symptoms are adynamic syndrome, with symptoms of malaise, fatigue, low grade fever, nausea and vomiting $(4,5)$. The particular newborn is that they progression of the inflammatory process frequently takes place without the presence of the characteristic septic temperatur. Cardiac activity has accelerated tones are muted; breathing is rapid, while the younger children appeared extreme tachypnea. The diagnosis of inflammation of the appendix begins with a physical examination, in which establishes the place and nature of pain. Difficulties arise when the atypical location, leading to the manifestation of symptoms resembling a number of other diseases of the adjacent organs $(4,18,19,24,25)$. There is no established specific indicator of clinical laboratory tests, which clearly indicates the presence of inflammation. Diagnosis occurs after an overall assessment of the research results of the blood count, ESR, biochemical indicators $\mathrm{AF}, \mathrm{C}$-reactive protein, urine $(6,14$, $15,20)$.

\section{Laboratory studies that may be helpful include the following:}

- Complete blood count (CBC)

- Measures of clotting function and coagulation parameters.

- Electrolyte levels

- Renal and liver function tests

- Etiology-specific serologic tests

- Urinalysis

- Tests for inflammatory markers and acute-phase reactants

- Culture of blood, urine, cerebrospinal fluid (CSF), or other tissues

Other studies that may be considered, depending on the clinical context, are as follows:

- Chest radiography

- Computed tomography (CT)

- Magnetic resonance imaging [MRI)

- Echocardiography.

- Lumbar puncture for CSF evaluation

Rapid recognition and diagnosis of sepsis is critical, and early goal-directed therapy can be very effective $(8,9,10)$. Relatively simple strategies have been used in the developing world to identify sepsis, and well-timed appropriate treatments, which include empirical antibiotics used in newborns and infants $(11,12,13)$ and early aggressive fluid resuscitation (14), have met with considerable success.

In view of the current definition of sepsis in children and infants where SIRS plus infection equals sepsis, it is crucially important to recognize systemic inflammatory response syndrome (SIRS) patients in everyday practice and to appreciate the importance of multifactorial diagnosis of SIRS, in accordance with the definition of systemic inflammatory response syndrome and peritonitis in children, was mostly confirmed by the combination of fever with respiratory rate $>2$ SD above normal for age $(76 \%)$. Fever with abnormal leukocyte count was present in 50\%, and fever with tachycardia $>2$ SD above normal for age in $15 \%$ (10-13). When children with leukocyte counts elevated or depressed for age but without temperature abnormalities were also included, only one additional child met the SIRS criteria.

\section{Sepsis diagnosis}

Sepsis, according to definition of the International consensus conference on pediatrics, was confirmed in $60 \%$ of cases by the combination of SIRS with suspected infection and in $40 \%$ of cases by the combination of SIRS with proven infection. Only $40 \%$ of the sepsis patients were treated in the ICU(13-15).

\section{Clinical and demographical data of SIRS and sepsis patients}

No gender difference was detected among the SIRS patients (boy/girl ratio 36/30 in the SIRS group) $(4,9,11)$. Median length of hospitalization was 7 days for SIRS patients (mean 11.3 days, range 2-62 days) and 9 days (mean16.2 days, range 4-44 days) for sepsis patients. Antibacterial therapy was used for $33 \%$ of SIRS patients $(7,9)$ and $100 \%$ of sepsis patients. In median antibacterial therapy was started on the $1^{\text {st }}$ day of hospitalization (on average $2^{\text {nd }}$, earliest on the 1 st day, latest on the $13^{\text {th }}$ day) in SIRS patients and on the $1^{\text {st }}$ day for all sepsis patients. The median duration of antibacterial therapy was 5 days in SIRS patients (mean 5.6 days, maximum 15 days) and 8 days in sepsis patients (8-9). Fatal cases were not established in this study. None of the SIRS and sepsis cases were recognized by doctors and recorded on the patients' cards (14, 16).

Several studies on children have established the prevalence of systemic inflammatory response syndrome (SIRS) and sepsis. We 
DIMCHEVA Y., et al.

used the National Library of Medicine (National Institutes of Health) database for a literature search and found a relatively low number of publications on this subject. We could find no publications describing the prevalence of SIRS among patients with abnormal temperatures in children population (17).

The prevalence of perforated appendicitis and SIRS among hospitalized children with fever was $72 \%$. Our literature review revealed one study reporting a $68 \%$ prevalence of SIRS in pediatric surgery units (15-17). SIRS affected one third of all hospitalized patients in the many studies (17). These data are not fully comparable with our data because different patient populations were studied.nagement of the potential sepsis sufferer. he presence of SIRS alone was not helpful in predicting the development of multiple organ dysfunction syndrome (MODS) since SIRS includes many nonprogressive conditions.

The same parameters that qualify patients for SIRS will also affect their category, which in turn affects work-up and final diagnosis. In addition, disposition may be driven not only by the results of history, physical examination, and supplemental testing, but also by the patient's initial presentation, including SIRS parameters for operating. Nonetheless, triage or "first recorded" vital signs have been used successfully as entry criteria in previous SIRS and sepsis research (16-17).

The effects of surgical stress, anesthesia, postoperative pain, and subsequent resuscitation may affect the components of the SIRS score. Critics have argued that SIRS scores, when calculated in the immediate postoperative period, may overestimate the proinflammatory response, making quantitation of SIRS at that time potentially too sensitive $(12,14,17)$.

\section{CONCLUSION}

Our results would indicate a high risk for sepsis development in children with SIRS. Early SIRS diagnosis and awareness of risk of developing sepsis could change the medical approach to the patient in everyday clinical practice, eventually leading to early, goaldirected therapy for sepsis. The duration of treatment of appendicitis complicated with syndrome systemic inflammatory response has an average of between 7 and 14 days. Early mobilization and enteral feeding period of accelerated postoperative recovery of the child. SIRS is a useful criterion for the recognition of postoperative complications and end-organ dysfunctions. Early recovery from SIRS may arrest the progression of organ dysfunction.

\section{REFERENCES}

1. Dellinger RP, Levy MM, Rhodes A. et al., Surviving Sepsis Campaign: International Guidelines for Management of Severe Sepsis and Septic Shock. Critical Care Medicine. 41: 580-637, 2013

2. Pavare J, Grope I, Gardovska D., Prevalence of systemic inflammatory response syndrome (SIRS) in hospitalized children: a point prevalence study. $B M C$ Pediatr. 9:25, 2009

3. Juskewitch JE, Prasad S, Salas CF, Huskins WC., Reliability of the identification of the systemic inflammatory response syndrome in critically ill infants and children. Pediatr Crit Care Med. 13(1):55-57, 2012

4. Chawla BK, Teitelbaum DH., Profound systemic inflammatory response syndrome following non-emergent intestinal surgery in children. J Pediatr Surg. 48:1936-40, 2013

5. Raines A, Garwe T, Wicks $\mathrm{R}$ et al.. Pediatric appendicitis: The prevalence of systemic inflammatory response syndrome upon presentation and its association with clinical outcomes. J Pediatr Surg. 48:24425,2013

6. Hsieh, C.H., Wang, Y.C. and Yang, H.R. Retroperitoneal abscess resulting from perforated acute appendicitis: analysis of its management and outcome. Surgery today, 37:762-767. 2007

7. Nichols, R.L., The treatment of intraabdominal infections in surgery. Diagnostic Microbiology and Infectious Disease, 12:195-199,1989

8. Bone, R.C. Balk, R.A. Cerra, F.B. et al, Definitions for sepsis and organ failure and guidelines for the use of innovative therapies in sepsis. The ACCP/SCCM Consensus Conference Committee. American College of Chest Physicians/Society of Critical Care Medicine. Chest, 101:1644-1655, 1992

9. Klempa, I. Current therapy of complicated appendicitis. Chirurg, 73:799-804. 2002

10.Nozoe, T., Matsumata, T. and Sugimachi, K., Significance of SIRS score in therapeutic strategy for acute appendicitis. Hepatogastroenterology, 49:444-446,2002

11.Calder, J.D. and Gajraj, H., Recent advances in the diagnosis and treatment of acute appendicitis. British Journal of Hospital Medicine, 54:129-133, 1995

12.Khristov, K., Bojcheva, A. and Kostova, S., Subphrenic abscess and suppurative pericarditis as complications in children 
with perforated appendicitis. Khirurgiia, 43:21-24, 1990

13.Vanamo, K. and Kiekara, O., Pylephlebitis after appendicitis in a child. European Journal of Pediatric Surgery, 36:15741576, 2001

14.Schultz, A., Jurgensen, P.M. and Jurgensen, S.P. Septic complications after appendicectomy for perforated appendicitis. A controlled clinical trial metronidazole and topical ampicillin. Acta Chir Scand, 149:517-520, 1983

15.Fanning, D.M., Barry, M. and O'Brien, G.C., Perforated retrocaecal appendix presenting as right lumbar abscess. Irish Journal of Medicine Sciences, 176:125128,2007
16.Mentefi, O., Zeybek, N. and Oysul, A. Stump appendicitis, rare complication after appendectomy: report of a case. Turkish Journal of Trauma \& Emergency Surgery, 14:330-332, 2008

17. Scott HF, Deakyne SJ, Woods JM, Bajaj L The prevalence and diagnostic utility of systemic inflammatory response syndrome vital signs in a pediatric emergency department. Acad Emerg Med; 22:381-9. 2015

18.Timothy Horeczko, Jeffrey P. Green, and Edward A. Panacek, Epidemiology of the Systemic Inflammatory Response Syndrome (SIRS) in the Emergency DepartmentWest J Emerg Med. ; 15: 329 336. 2014 